\title{
EXPONENTIAL STABILIZATION OF CASCADE ODE-LINEARIZED KDV SYSTEM BY BOUNDARY DIRICHLET ACTUATION
}

\author{
HABIB AYADI
}

\begin{abstract}
In this paper, we solve the problem of exponential stabilization for a class of cascade ODE-PDE system governed by a linear ordinary differential equation and a $1-d$ linearized Korteweg-de Vries equation $(\mathrm{KdV})$ posed on a bounded interval. The control for the entire system acts on the left boundary with Dirichlet condition of the KdV equation whereas the $\mathrm{KdV}$ acts in the linear ODE by a Dirichlet connection. We use the socalled backstepping design in infinite dimension to convert the system under consideration into an exponentially stable cascade ODE-PDE system. Then, we use the invertibility of such design to achieve the exponential stability for the ODE-PDE cascade system under consideration by using Lyapunov analysis.
\end{abstract}

\section{CONTENTS}

1. Introduction

2. Problem Formulation and Main Result

3. Control Design

4. Proof of Theorem 2.1

4.1. First step: Invertibility of $\Omega$

4.2. Second step: Well Posedness

4.3. Third step: Exponential Stability

References

\section{INTRODUCTION}

It is well known that the Korteweg-de Vries (KdV) equation in bounded domain models the dynamics of various types of extreme waves in shallow water, more particularly tsunami waves and freak waves. From theoretical point of view, the KdV controlled equation has

2010 Mathematics Subject Classification. 93D05, 93D20, 34D05, 34D20.

Key words and phrases. Cascade ODE-PDE, Linearized-KdV, Backstepping, Exponential stability. 
some interesting control properties depending on where the controls are located [8], [20]. In the past decades, stabilization of coupled ODE-PDE systems is widely studied in the literature. Such systems can be used to model various processes such as road traffic [11], gas flow pipeline [10], power converters connected to transmission lines [9], oil drilling [4] and many other systems. Many problems of state and output feedback stabilization for coupled ODE-Heat has been solved [21], [22], [14] and ODE- Wave [13], [23], to cite few. The problem of controllability of coupled ODE-PDE systems has been discussed in [25] and [26]. Some nonlinear extensions are studied in [24], [2], [6] where the non linearity is assumed to be global Lipshitz, and in [5], [1], [7] for more general nonlinear ODE. In this paper, we deal with the state stabilization problem for a cascade ODE-KdV system. We applied the infinite dimensional backstepping method to build a stabilizing feedback control for system (1)-(6). The backstepping method was introduced firstly for finite dimensional control systems governed by ODE [12]. The first extensions to PDE have appeared in [3] and [17]. Later, in [19] and [15], the authors have introduced an invertible integral transformation that transforms the original parabolic PDE into an asymptotically stable one. Recently, the backstepping method is used to design a feedback control law for coupled PDE-ODE (see the textbook [16] and references therein). As far as we know, problem of stabilization by backstepping design for such system when the PDE subsystem is governed by the linearized $\mathrm{KdV}$ equation has not yet been tackled in the literature. This paper is organized as follows. In Section 2, we present the main result of this paper which is summarized in Theorem 2.1. In section 3, we formulate the backstepping design of the feedback control law. Section 4 is devoted to prove Theorem 2.1 through three steps as follows. Firstly, we prove the invertibility of the transformation given in previous section. Secondly, we establish the well posedness of system (1)-(6). Finally, we prove the exponential stability in the sens of the $H$-norm of solutions of (1)-(6) around the origin. 


\section{Problem Formulation and Main Result}

Let $l>0$, we consider the following cascade ODE-PDE system

$$
\begin{gathered}
\dot{X}(t)=A X(t)+B u(l, t), \quad t>0, \\
u_{t}(x, t)=-u_{x}(x, t)-u_{x x x}(x, t), \quad t>0, \quad x \in(0, l), \\
u(0, t)=U(t), \quad t>0, \\
u_{x}(l, t)=0, \quad t>0, \\
u_{x x}(l, t)=0, \quad t>0 . \\
X(0)=X_{0}, u(x, 0)=u_{0}(x), \quad x \in(0, l) .
\end{gathered}
$$

where $X(t) \in \mathbb{R}^{n}$ is the state of the ODE subsystem, $u(x, t) \in \mathbb{R}$ is the state of the linear KdV subsystem, $U(t) \in \mathbb{R}$ is the control input to the entire system acting in the left boundary $x=0$ of the PDE domain $(0, l)$, and $A \in \mathbb{R}^{n \times n}, B \in \mathbb{R}^{n \times 1}$ such that the pair $(A, B)$ is controllable. The control objective is to exponentially stabilize the system (1)- (6) around its zero equilibrium. Along this paper, the Euclidean norm of a vector $X$ in $\mathbb{R}^{n}$ and the $L^{2}$-norm of a function $u$ in $L^{2}(0, l)$ are denoted by $|X|$ and

$$
\|u\|=\left(\int_{0}^{l} u^{2}(x) d x\right)^{\frac{1}{2}}
$$

respectively. Let $H=\mathbb{R}^{n} \times L^{2}(0, l)$ the state space of the system (1)-(6). It is obvious that the vector space $H$ equipped with its norm

$$
\|(X, u)\|_{H}=\left(|X|^{2}+\|u\|^{2}\right)^{\frac{1}{2}}
$$

is a Hilbert space. The infinite dimensional backstepping design for coupled ODE-PDE system is to seek a continuous and invertible integral transformation

$$
\Omega \quad: \quad H \rightarrow H
$$

$$
(X, u) \mapsto(X, w)
$$


to convert system (1)-(6) into the exponentially stable target system

$$
\begin{gathered}
\dot{X}(t)=(A+B K) X(t)+B w(l, t), \quad t>0, \\
w_{t}(x, t)=-w_{x}(x, t)-w_{x x x}(x, t)-\lambda w(x, t), \quad t>0, \quad x \in(0, l), \\
w(0, t)=0, \quad t>0, \\
w_{x}(l, t)=0, \quad t>0, \\
w_{x x}(l, t)=0, \quad t>0 . \\
X(0)=X_{0}, w(x, 0)=w_{0}, \quad x \in(0, l),
\end{gathered}
$$

where $K \in \mathbb{R}^{1 \times n}$ such that $A+B K$ is Hurwitz, and $\lambda$ is an arbitrary positive number. Define the dense subspace $\Lambda$ of $H$ by

$$
\Lambda=\mathbb{R}^{n} \times\left\{u \in H^{3}(0, l) \mid u(0)=u^{\prime}(l)=u^{\prime \prime}(l)=0\right\} .
$$

Our main result is stated in the following theorem which asserts that the cascade ODE-PDE system (1)-(6) is exponentially stable in the sense of the norm $\|\cdot\|_{H}$.

Theorem 2.1. For any initial condition $\left(X_{0}, u_{0}\right) \in \Lambda$, the closed loop system (1)-(6) with the feedback law (31) admits a unique classical solution

$$
(X, u) \in C([0,+\infty), \Lambda) \cap C^{1}([0,+\infty) ; H)
$$

and if $\left(X_{0}, u_{0}\right) \in H$ then the system admits a unique mild solution

$$
(X, u) \in C([0,+\infty), H) .
$$

Moreover, there exists two constants $c_{1}>0$ and $c_{2}>0$ such that for all $\left(X_{0}, u_{0}\right) \in H$ then

$$
\|(X(t), u(., t))\|_{H} \leq c_{1}\left\|\left(X_{0}, u_{0}\right)\right\|_{H} e^{-c_{2} t}, \forall t \geq 0 .
$$

\section{Control Design}

The transformation $\Omega$ is postulated in the following form :

$$
\begin{aligned}
X(t) & =X(t), \\
w(x, t) & =u(x, t)-\int_{x}^{l} u(y, t) q(x, y) d y-\varphi(x) X(t),
\end{aligned}
$$


where the kernel $q(x, y) \in \mathbb{R}$ and the gain function $\varphi(x)^{T} \in \mathbb{R}^{n}$ are to be determined. From 177, the first three derivatives of $w(x, t)$ with respect to $x$ are given by

$$
\begin{aligned}
& w_{x}(x, t)=u_{x}(x, t)+q(x, x) u(x, t)-\int_{x}^{l} u(y, t) q_{x}(x, y) d y-\varphi^{\prime}(x) X(t), \\
& w_{x x}(x, t)=u_{x x}(x, t)+u(x, t) \frac{d}{d x} q(x, x)+q(x, x) u_{x}(x, t)+q_{x}(x, x) u(x, t)-\int_{x}^{l} u(y, t) q_{x x}(x, y) d y \\
& \begin{aligned}
&(19)-\varphi^{\prime \prime}(x) X(t) \\
& \text { and, } w_{x x x}(x, t)= u_{x x x}(x, t)+u(x, t) \frac{d^{2}}{d x^{2}} q(x, x)+2 u_{x}(x, t) \frac{d}{d x} q(x, x)+q(x, x) u_{x x}(x, t)+u(x, t) \frac{d}{d x} q_{x}(x, x) \\
& \text { (20) }+q_{x}(x, x) u_{x}(x, t)+q_{x x}(x, x) u(x, t)-\int_{x}^{l} u(y, t) q_{x x x}(x, y) d y-\varphi^{\prime \prime \prime}(x) X(t),
\end{aligned}
\end{aligned}
$$

respectively. Furthermore, the derivative of (17) with respect to $t$ is given by

$$
\begin{aligned}
w_{t}(x, t) & =u_{t}(x, t)-\int_{x}^{l} u_{t}(y, t) q(x, y) d y-\varphi(x) \dot{X}(t), \\
& =u_{t}(x, t)+\int_{x}^{l}\left(u_{y}(y, t)+u_{y y y}(y, t)\right) q(x, y) d y-\varphi(x)(A X(t)+B u(l, t)) .
\end{aligned}
$$

Integrating by parts the above identity, we get

$$
\begin{aligned}
w_{t}(x, t)= & u_{t}(x, t)-\int_{x}^{l} u(y, t)\left(q_{y}(x, y)+q_{y y y}(x, y)\right) d y-q(x, x)\left(u(x, t)+u_{x x}(x, t)\right) \\
& +q_{y}(x, x) u_{x}(x, t)-q_{y y}(x, x) u(x, t)+\left(q(x, l)+q_{y y}(x, l)\right) u(l, t) \\
& -\varphi(x)(A X(t)+B u(l, t)),
\end{aligned}
$$

where the boundary conditions (4) and (6) has been used. Thus, from (17), (18), (20) and (22), for all $\lambda>0$, the identity

$$
\begin{aligned}
w_{t}(x, t)+w_{x}(x, t)+w_{x x x}+\lambda w(x, t)= & -\int_{x}^{l} u(y, t)\left(q_{x x x}(x, y)+q_{y y y}(x, y)+q_{x}(x, y)+q_{y}(x, y)+\lambda q(x, y)\right) d y \\
& +\left(q(x, l)+q_{y y}(x, l)-\varphi(x) B\right) u(l, t) \\
& +u_{x}(x, t)\left(q_{y}(x, x)+q_{x}(x, x)+2 \frac{d}{d x} q(x, x)\right) \\
& +u(x, t)\left(\lambda+q_{x x}(x, y)+\frac{d}{d x} q_{x}(x, x)+\frac{d^{2}}{d x^{2}} q(x, x)\right) \\
& -\left(\varphi(x)\left(A+\lambda I_{n}\right)+\varphi^{\prime \prime}(x)+\varphi^{\prime \prime \prime}(x)\right) X(t),
\end{aligned}
$$


holds. Moreover, setting $x=0$ in (17) and $x=l$ in (17), (18) and (19), it follows

$$
\begin{aligned}
w(0, t) & =U(t)-\int_{0}^{l} q(0, y) u(y, t) d y-\varphi(0) X(t), \\
w(l, t) & =u(l, t)-\varphi(l) X(t), \\
w_{x}(l, t) & =q(l, l) u(l, t)-\varphi^{\prime}(l) X(t), \\
w_{x x}(l, t) & =\frac{d q_{x}}{d x}(l, l) u(l, t)+q_{x}(l, l) u(l, t)-\varphi^{\prime \prime}(l) X(t) .
\end{aligned}
$$

Assume that the gain function $\varphi(x)^{T}$ defined in $[0, l]$ and the kernel $q(x, y)$ defined in the triangle

$$
T=\{(x, y) \mid x \in[0, l], y \in[x, l]\}
$$

satisfy

$$
\begin{aligned}
\varphi^{\prime \prime \prime}(x)+\varphi^{\prime}(x)+\varphi(x)\left(A+\lambda I_{n}\right) & =0, \quad x \in[0, l], \\
\varphi(l) & =K, \\
\varphi^{\prime}(l) & =0, \\
\varphi^{\prime \prime}(l) & =0,
\end{aligned}
$$

and

$$
\begin{aligned}
q_{x x x}(x, y)+q_{y y y}(x, y)+q_{x}(x, y)+q_{y}(x, y) & =-\lambda q(x, y), \quad(x, y) \in T, \\
q(x, l)+q_{y y}(x, l) & =\varphi(x) B, \quad x \in[0, l], \\
q(x, x) & =0, \quad x \in[0, l], \\
q_{x}(x, x) & =\frac{\lambda}{3}(l-x), \quad x \in[0, l],
\end{aligned}
$$

respectively. Then, from the identities (23)-(27), we obtain the target system (9)-(14) for every solution of the closed loop (10)-(6) with the feedback law

$$
U(t)=\int_{0}^{l} q(0, y) u(y, t) d y+\varphi(0) X(t) .
$$

Notice that the existence of the control law (31) is a direct consequence of the existence of kernels $q(x, y)$ and $\varphi(x)^{T}$ that satisfy (30) and [29), respectively. In the following, we compute explicitly the kernel $\varphi(x)^{T}$ and we prove the existence of the kernel $q(x, y)$. To begin with, the solution of the ODE (29) is

$$
\varphi(x)=(K, 0,0) e^{(x-l) M} E,
$$


where $M$ and $E$ are the constant matrices

$$
M=\left(\begin{array}{ccc}
0 & 0 & -\left(A+\lambda I_{n}\right) \\
I_{n} & 0 & -I_{n} \\
0 & I_{n} & 0
\end{array}\right), E=\left(\begin{array}{l}
I_{n} \\
0 \\
0
\end{array}\right)
$$

On the other hand, to prove the existence of the kernel $q(x, y)$, let us make the change of variables

$$
s=x+y \quad, \quad t=y-x,
$$

and define

$$
G(s, t):=q(x, y)=q\left(\frac{s-t}{2}, \frac{s+t}{2}\right)
$$

Then, the function $G$ defined in the triangle $T_{0}=\{(s, t) / t \in[0, l], s \in[t, 2 l-t]\}$, satisfies

$$
\begin{aligned}
\left(6 G_{t t s}+2 G_{s s s}+2 G_{s}\right)(s, t) & =-\lambda G(s, t), \quad(s, t) \in T_{0}, \\
\left(G+G_{s s}+2 G s t+G_{t t}\right)(s, 2 l-s) & =\varphi(s-l) B, \quad s \in[l, 2 l], \\
G(s, 0) & =0, \quad s \in[0,2 l], \\
G_{t}(s, 0) & =\frac{\lambda}{6}(s-2 l), \quad s \in[0,2 l] .
\end{aligned}
$$

To prove the existence of the kernel $q(x, y)$, we adopt the proof done by [8, Cerpa-Coron] with slight modification. At this stage, it is conventional to put the previous system in an integral form (see for example [8]). Using (34), we rewrite (33) in variable $(\eta, \xi)$ as follows

$$
\left(6 G_{t t s}+6 G_{s s s}+12 G_{s t s}+6 G_{s}\right)(\eta, \xi)=\left(-\lambda G+4 G_{s s s}+12 G_{s t s}+4 G_{s}\right)(\eta, \xi) .
$$

Next, we integrate (37) with respect to the variable $\eta$ over the interval $(s, 2 l-\xi)$ and use the boundary condition (34). Then, we integrate the obtained identity with respect to $\xi$ over the interval $(0, \tau)$ and use (35) and 36. Finally, by integrating the acquired identity with respect to $\tau$ over the interval $(0, t)$ and use again the boundary condition $(35)$, it follows

$$
\begin{gathered}
G(s, t)=\int_{0}^{t} \int_{0}^{\tau} \varphi(l-\xi) B d \xi d \tau-\frac{\lambda t}{6}(2 l-s)-2 \int_{0}^{t} G_{s}(s, \tau) d \tau-\int_{0}^{t} \int_{0}^{\tau}\left(G_{s s}+G\right)(s, \xi) d \xi d \tau \\
\text { (38) }-\frac{1}{6} \int_{0}^{t} \int_{0}^{\tau} \int_{s}^{2 l-\xi}\left(4 G_{s s s}+4 G_{s}-\lambda G+12 G_{s t s}\right)(\eta, \xi) d \eta d \xi d \tau .
\end{gathered}
$$


In order to achieve the existence of the function $G$, we use the classical method of successive approximations [18]. To this end, set

$$
\begin{aligned}
G^{1}(s, t)= & -\frac{\lambda t}{6}(2 l-s) \\
G^{n+1}(s, t)= & \theta(t)-2 \int_{0}^{t} G_{s}^{n}(s, \tau) d \tau-\int_{0}^{t} \int_{0}^{\tau}\left(G_{s s}^{n}+G^{n}\right)(s, \xi) d \xi d \tau \\
& -\frac{1}{6} \int_{0}^{t} \int_{0}^{\tau} \int_{s}^{2 l-\xi}\left(4 G_{s s s}^{n}+4 G_{s}^{n}-\lambda G^{n}+12 G_{s t s}^{n}\right)(\eta, \xi) d \eta d \xi d \tau
\end{aligned}
$$

where

$$
\theta(t)=\int_{0}^{t} \int_{0}^{\tau} \varphi(l-\xi) B d \xi d \tau
$$

Using an appropriate calculation, we get

$$
G^{2}(s, t)=\theta(t)+\frac{1}{6}\left(-\lambda t^{2}+\left(\frac{\lambda l}{9}-\frac{\lambda^{2} l^{2}}{18}\right) t^{3}+\frac{\lambda}{18} t^{4}+\frac{\lambda^{2}}{240} t^{5}+\left(\frac{l \lambda^{2}-\lambda}{18}\right) s t^{3}-\frac{\lambda^{2}}{72} s^{2} t^{3}\right) .
$$

Since $\varphi$ is a continuous function on $[0, l]$, from $[40$, there exists a constant $\rho>0$ such that $|\theta(t)| \leq \rho t^{2}$. Keeping this in mind, and using the classical procedure as in [8], we get the following inequality

$$
\left|G^{k}(s, t)\right| \leq \sum_{i=0}^{k}\left(\sum_{j=\left[\frac{3 k-1}{2}\right]-i}^{3 k-1-i} a_{i j} t^{j}\right) s^{i},
$$

for all $k \in \mathbb{N}^{*}$, where the positive coefficients $a_{i j}$ have appropriate decay properties so that the series $\sum_{n=1}^{\infty} G^{n}(s, t)$ is uniformly convergent in $T_{0}$. We underline that the uniform convergence of the previous series on the domain $T_{0}$ is due to the fact that the recursive identity is a function of some integral operators. Therefore, the series defines a continuous function

$$
G(s, t)=\sum_{n=1}^{\infty} G^{n}(s, t), \forall(s, t) \in T_{0} .
$$

Thus, the proof of the existence of $G(s, t)$ solution of the integral equation (38) is achieved. Having proved the existence of $G(s, t)$ in $T_{0}$, that of $q(x, y)$ in $T$ follows immediately. In consequence, the state feedback controller 31 is well defined.

Let's move to the proof of the main Theorem 2.1.

\section{Proof of Theorem 2.1}

The proof is divided in three steps. In the first step, we prove the invertibility of the backstepping transformation $\Omega$ defined by $(8)$. The existence and the global exponential 
stability of the solution to the closed loop system (1)-(6) with the state feedback (31) are established in the second and the third step, respectively.

4.1. First step: Invertibility of $\Omega$. It is a straightforward that the transformation $\Omega$ is invertible, and

$$
\begin{aligned}
\Omega^{-1}: & H \rightarrow H \\
& (X, w) \mapsto(X, u)
\end{aligned}
$$

has the following form

$$
\begin{aligned}
X(t) & =X(t) \\
u(x, t) & =w(x, t)+\int_{x}^{l} w(y, t) h(x, y) d y+\psi(x) X(t),
\end{aligned}
$$

where the kernel function $h(x, y) \in \mathbb{R}$ and the gain function $\psi(x)^{T} \in \mathbb{R}^{n}$ are to be determined. As is done in the study of the direct transformation $\Omega$, the derivatives $u_{t}, u_{x}, u_{x x x}$ are computed and system (1)-(6) holds if $h(x, y)$ satisfies

$$
\begin{aligned}
h_{x x x}(x, y)+h_{y y y}(x, y)+h_{x}(x, y)+h_{y}(x, y) & =\lambda h(x, y), \\
h(x, l)+h_{y y}(x, l) & =-\psi(x) B, \\
h(x, x) & =0, \\
h_{x}(x, x) & =\frac{\lambda}{3}(l-x),
\end{aligned}
$$

in the triangle $T$ defined by $(28)$ and $\psi(x)$ satisfies in $[0, l]$

$$
\begin{array}{r}
\psi^{\prime \prime \prime}(x)+\psi^{\prime \prime}(x)+\varphi(x)(A+B K)=0, \\
\psi(l)=K, \\
\psi^{\prime}(l)=0, \\
\varphi^{\prime \prime}(l)=0 .
\end{array}
$$

First of all, the solution of the ODE (45) is

$$
\psi(x)=(K, 0,0) e^{(x-l) N} E,
$$


where $N$ and $E$ are two constant matrices given by

$$
N=\left(\begin{array}{ccc}
0 & 0 & -(A+B K) \\
I_{n} & 0 & -I_{n} \\
0 & I_{n} & 0
\end{array}\right), E=\left(\begin{array}{l}
I_{n} \\
0 \\
0
\end{array}\right) .
$$

Then, in the same way as for the kernel $q(x, y)$, one can easily prove the existence and the continuity of the kernel $h(x, y)$ in $T$. Moreover, $\Omega^{-1}$ is continuous operator on the Hilbert space $H$. This achieves the first step of the proof of Theorem 2.1

4.2. Second step: Well Posedness. Since $\Omega: H \rightarrow H$ is a continuous invertible transformation, $\Omega^{-1}$ maps a trajectory of (9)-(14) into a trajectory of (1)-(6) with the state feedback controller (31). Hence, to prove that (1)-(6) is well posed, it suffices to establish the well posedness of the target system (9)-(14). To this reason, we consider the target KdV subsystem (10)-14) in the Hilbert state space $L^{2}(0, l)$ and, we define the unbounded linear operator $\Gamma: \mathscr{D}(\Gamma) \rightarrow L^{2}(0, l)$ by

$$
\Gamma(w)=-\lambda w-w^{\prime}-w^{\prime \prime \prime}
$$

with domain $\mathscr{D}(\Gamma)=\left\{w \in H^{3}(0, l) \mid w(0)=w^{\prime}(l)=w^{\prime \prime}(l)=0\right\}$. A simple computation shows that the adjoint operator $\Gamma^{*}$ of $\Gamma$ is

$$
\Gamma^{*}(w)=-\lambda w+w^{\prime}+w^{\prime \prime \prime},
$$

with domain $\mathscr{D}\left(\Gamma^{*}\right)=\left\{w \in H^{3}(0, l) \mid w(0)=w^{\prime}(0)=0, w(l)+w^{\prime \prime}(l)=0\right\}$ It is obvious that the domains $\mathscr{D}(\Gamma)$ and $\mathscr{D}\left(\Gamma^{*}\right)$ are dense subspaces in $L^{2}(0, l)$. Moreover, by integrations by parts, we get

$$
\begin{aligned}
& <\Gamma(w), w>=-\frac{1}{2}\left(w(l)^{2}+w^{\prime}(0)^{2}\right)-\lambda\|w\|^{2} \leq 0, \forall w \in \mathscr{D}(\Gamma), \\
& <\Gamma^{*}(v), v>=-\frac{1}{2}\left(v(l)^{2}+v^{\prime}(l)^{2}\right)-\lambda\|v\|^{2} \leq 0, \forall v \in \mathscr{D}\left(\Gamma^{*}\right),
\end{aligned}
$$

where $<,>$ stands for the $L^{2}(0, l)$ standard inner product. Thus, both operators $\Gamma$ and $\Gamma^{*}$ are dissipative. Therefore, according to the Lumer-Phillips Theorem's, the operator $\Gamma$ generates a $C^{0}$ semigroup of contraction in $L^{2}(0, l)$. Consequently, for all initial condition $w_{0} \in \mathscr{D}(\Gamma)$, the system (10)-(14) has a unique classical solution

$$
w \in C([0,+\infty) ; \mathscr{D}(\Gamma)) \cap C^{1}\left([0,+\infty) ; L^{2}(0, l)\right) .
$$


Furthermore, by Duhamel formula's, for all initial condition $X_{0} \in \mathbb{R}^{n}$, the ODE (9) has a unique global solution

$$
X(t)=e^{t(A+B K)} X_{0}+\int_{0}^{t} e^{(t-\tau)(A+B K)} B w(l, \tau) d \tau
$$

We conclude that the target system (91-14) is well posed in $H$, and for all initial condition $\left(X_{0}, w_{0}\right) \in \Lambda$, system (9)-(14) has a unique classical solution

$$
(X, w) \in C([0,+\infty) ; \Lambda) \cap C^{1}([0,+\infty) ; H),
$$

and for $\left(X_{0}, w_{0}\right) \in H$, the system (9)-14) has a unique mild solution

$$
(X, w) \in C([0,+\infty) ; H) .
$$

4.3. Third step: Exponential Stability. Consider the Lyapunov function candidate

$$
V(t)=X(t)^{T} P X(t)+\frac{\mu}{2}\|w(., t)\|_{L^{2}(0, l)}^{2},
$$

where $\mu>0$ is a constant to be designed later and the positive definite matrix $P=P^{T}>0$ is the solution of the Lyapunov equation

$$
P(A+B K)+(A+B K)^{T} P=-Q,
$$

for some positive definite matrix $Q=Q^{T}>0$. From 499, it can be obtained that for all $t \geq 0$,

$$
\alpha_{1}\|(X(t), w(., t))\|_{H}^{2} \leq V(t) \leq \alpha_{2}\|(X(t), w(., t))\|_{H}^{2},
$$

where

$$
\alpha_{1}=\min \left(\lambda_{\min }(P), \frac{\mu}{2}\right) \text { and } \alpha_{2}=\max \left(\lambda_{\max }(P), \frac{\mu}{2}\right) .
$$

The derivative of $V$ along the solutions of (9)-(14) is given by

$$
\dot{V}(t)=-X^{T}(t) Q X(t)+2 X(t)^{T} P B w(l, t)-\mu w(l, t)^{2}-\mu \lambda\|w(., t)\|^{2} .
$$

By Young's inequality, we get

$$
2 X(t)^{T} P B w(l, t) \leq \frac{\lambda_{\min }(Q)}{2}|X(t)|^{2}+\frac{2}{\lambda_{\min }(Q)}|P B|^{2} w(l, t)^{2} .
$$

Thus,

$$
\dot{V}(t) \leq-\frac{\lambda_{\min }(Q)}{2}|X(t)|^{2}-\left(\mu-\frac{2}{\lambda_{\min }(Q)}|P B|^{2}\right) w(l, t)^{2}-\mu \lambda\|w(., t)\|^{2} .
$$


Now, using (50) and choose

$$
\mu>\operatorname{Max}\left(\frac{2}{\lambda_{\min }(Q)}|P B|^{2}, 2 \lambda_{\max }(P)\right) .
$$

Then, the inequality

$$
\dot{V}(t) \leq-\delta V(t),
$$

holds for all $t \geq 0$, where $\delta=\frac{\min \left(\lambda_{\min }(Q), 4 \lambda\right)}{\mu}$. Therefore,

$$
V(t) \leq V(0) e^{-\delta t}, \forall t \geq 0 .
$$

Using (50) and (52), by tacking $\alpha=\frac{\alpha_{2}}{\alpha_{1}}$, it follows that

$$
\|(X(t), w(., t))\|_{H}^{2} \leq \alpha\left\|\left(X_{0}, w_{0}\right)\right\|_{H}^{2} e^{-\delta t}, \forall t \geq 0 .
$$

Recall that the transformations $\Omega$ and $\Omega^{-1}$ are linear and continuous, then there exist two positive constants $d_{1}$ and $d_{2}$ such that

$$
\begin{gathered}
\|(X, w)\|_{H}=\|\Omega(X, u)\|_{H} \leq d_{1}\|(X, u)\|_{H}, \\
\|(X, u)\|_{H}=\left\|\Omega^{-1}(X, w)\right\|_{H} \leq d_{2}\|(X, w)\|_{H} .
\end{gathered}
$$

Hence, for all initial condition $\left(X_{0}, u_{0}\right) \in H$, for all $t \geq 0$, we obtain from (53), 554) and (55)

$$
\|(X(t), u(., t))\|_{H} \leq c_{1}\left\|\left(X_{0}, u_{0}\right)\right\|_{H} e^{-c_{2} t},
$$

where $c_{1}=d_{1} d_{2} \sqrt{\alpha}$ and $c_{2}=\frac{\delta}{2}$. Thus, the proof of Theorem 2.1 is complete.

Remark 4.1. Since the positive definite matrix $Q$ and the positive parameter $\lambda$ are arbitrary, the decay rate $c_{2}$ in (15) is arbitrary. Thus, the system (17)-(6) is rapidly exponentially stable.

\section{REFERENCES}

[1] O. M. Aamoa, A. Hasana and M. Krstic, Boundary observer design for hyperbolic PDE-ODE cascade systems, Automatica, 68 (2016), 75-86

[2] T. Ahmed-Ali, F. Giri, M. Krstic and F. Lamnabhi-Lagarrigue, Observer design for a class of nonlinear ODE-PDE cascade systems, Systems \& Control Letters, 83 (2015), 19-27.

[3] B. D'Andréa Novel and J.-M. Coron, Stabilization of a rotating body beam without damping, IEEE Transaction on Automatic Control, 43 (1998), 608-618.

[4] J. C. Avila Vilchis, S. Mondié and B. Saldivar, The Control of Drilling Vibrations: A Coupled PDE-ODE Modeling Approach, International Journal Applied Mathematics Computer Science, 26 (2016), 335-349. 
[5] N. Bekiaris-Liberis and M. Krstic, Compensation of Wave Actuator Dynamics for Nonlinear Systems, IEEE Transaction on Automatic Control, 59 (2014), 1555-1569.

[6] X. Cai, L. Liao, J. Zhang and W. Zhang, Observer design for a class of nonlinear system in cascade with counter-convecting transport dynamics, Kybernetika, 52 (2016), 76-88.

[7] X. Cai and M. Krstic, Nonlinear stabilization through wave PDE dynamics with a moving uncontrolled boundary, Automatica, 68 (2016), 27-38.

[8] E. Cerpa and J.-M. Coron, Rapid stabilization for a Korteweg-de Vries equation from the Left dirichlet boundary condition, IEEE transaction on automatic control, 58 (2013), 1688-1695.

[9] J. Daafouz, M. Tucsnak and J. Valein, Nonlinear control of a coupled PDE-ODE system modeling a switched power converter with a transmission line, Systems \& Control Letters, 70 (2014), 92-99.

[10] A. Girard, C. Prieur and Y. Tang, Stability analysis of a singularly perturbed coupled ODE-PDE system, IEEE Conference on Decision and Control (2015).

[11] P. Goatin, The Aw-Rascle vehicular traffic flow model with phase transitions, Mathematical and Computer Modelling, 44 (2006), 287-303.

[12] I. Kanellakopoulos, P. Kokotovic and M. Kristic, Nonlinear and Adaptive Control Design, John Wiley and Sons, (1995).

[13] M. Krstic and G. A. Susto, Control of PDE-ODE cascades with Neumann interconnections, Journal of Franklin Institute, 347 (2010), 284-314.

[14] M. Krstic, Compensating actuator and sensor dynamics governed by diffusion PDEs, Systems \& Control Letters, 58 (2009), 372-377.

[15] M. Krstic and A. Smyshlyaev, Closed-form boundary state feedbacks for a class of $1-d$ partial integrodifferential equations, IEEE Transaction on Automatic Control, 49 (2004), 2185-2202.

[16] M. Krstic, Delay Compensation for Nonlinear, Adaptive, and PDE Systems, Birkhauser, (2009).

[17] M. Krstic and W. Liu, Backstepping boundary control of Burgers equation with actuator dynamics, Systems \& Control Letters, 41 (2000), 291-303.

[18] M. Krstic and A. Smyshlyaev, Boundary Control of PDEs: A Course on Backstepping Designs, SIAM, (2008).

[19] W. Liu, Boundary feedback stabilization of an unstable heat equation, SIAM Journal Control Optimization, 42 (2003), 1033-1043.

[20] L. Rosier, Exact boundary controllability for the Korteweg-de Vries equation on a bounded domain, ESAIM Control Optim. Calc. Var., 2 (1997), 33-55.

[21] S. Tang and C. Xie, Stabilization for a coupled PDE-ODE control system, Journal of the Franklin Institute, 348 (2011), 2142-2155.

[22] S. Tang and C. Xie, State and output feedback boundary control for a coupled PDE-ODE system. Systems \& Control Letters, 60 (2011), 540-545.

[23] S. Tang and Z. Zhoua, Boundary stabilization of a coupled wave-ODE system with internal anti-damping, International Journal of Control, 85 (2012), 1683-1693.

[24] J. W. Wang and H. N. Wu, Observer design and output feedback stabilization for nonlinear multivariable systems with diffusion PDE-governed sensor dynamics, Nonlinear Dynamics, 72 (2013), 615-628. 
[25] G. Weiss and X. Zhao, Well-posedness and Controllability of a Class of Coupled Linear Systems, SIAM Journal on Control and Optimization, 48 (2009), 2719-2750.

[26] G. Weiss and X. Zhao, Controllability and Observability of a Well-posed System Coupled with a Finitedimensional System, IEEE Transactions on Automatic Control, 56 (2011), 1-12.

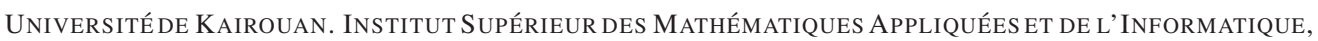
Avenue Assad Iben Fourat - 3100 Kairouan, Tunisia

E-mail address: hayadi26@laposte.net 